\title{
ESTIMATIVA DA INCERTEZA DE MEDIÇÃO: ESTUDO DE CASO NO ENSAIO DE MIGRAÇÃO DE $\varepsilon$-CAPROLACTAMA COM DETERMINAÇÃO POR CG-DIC
}

\author{
Fabio Silvestre Bazilio*, Marcus Vinícius Justo Bomfim, Rodrigo Justo de Almeida e Shirley de Mello Pereira Abrantes \\ Departamento de Química, Instituto Nacional de Controle de Qualidade em Saúde, Fundação Oswaldo Cruz, Av. Brasil, 4365, \\ 21040-900 Rio de Janeiro - RJ, Brasil
}

Recebido em 11/11/11; aceito em 14/2/12; publicado na web em 30/4/12

\begin{abstract}
ESTIMATION OF MEASUREMENT UNCERTAINTY: A CASE STUDY OF THE MIGRATION TEST OF $\varepsilon$-CAPROLACTAM WITH DETERMINATION BY GC-FID. The estimation of measurement uncertainty of an analytical result is an important tool to be applied for compliance to ISO IEC 17025. Through the available guides it is possible to establish procedures and criteria for the estimation of measurement uncertainty. This paper presents a case study on the estimation of measurement uncertainty in migration the test of $\varepsilon$-caprolactam from the polyamide 6 packages to fatty foods with determination by GC-FID. The calculation methodology used for the estimation of measurement uncertainty for the migration test was developed based on the guides EURACHEM / CITAC and ISO GUM, taking into account relevant aspects of the migration test. The expanded uncertainty estimated was approximately $23 \%$ of the concentration of migration.
\end{abstract}

Keywords: measurement uncertainty; migration analysis; gas chromatography.

\section{INTRODUÇÃO}

A $\varepsilon$-caprolactama é um monômero utilizado na reação de polimerização da poliamida 6 , utilizada como embalagem de alimentos resfriados, como blanquet de peru, mortadela, presunto, entre outros. Após a polimerização, parte do monômero pode permanecer na embalagem e migrar para os alimentos durante o tempo de acondicionamento. ${ }^{1}$ A Resolução no 105 , de 19/5/1999, da Agência Nacional de Vigilância Sanitária - ANVISA estabelece limite de migração específica de $\varepsilon$-caprolactama em alimento ou solvente simulante em $15 \mathrm{mg} \mathrm{kg}^{-1}$ ou $15 \mathrm{mg} \mathrm{L}^{-1}$. ${ }^{2}$ Sendo este um limite de decisão, onde uma determinada amostra pode ser reprovada ou aprovada de acordo com o resultado analítico, é importante que a metodologia de análise empregada seja capaz de produzir resultados adequados e confiáveis.

Com o objetivo de adequar o ensaio às normas de qualidade de forma a garantir a confiabilidade dos resultados analíticos e obter o reconhecimento da competência do laboratório, um importante passo a ser dado é a acreditação do ensaio, segundo os requisitos da norma ABNT NBR ISO/IEC 17025:2005. Tal norma estabelece requisitos gerais para a competência de laboratórios de ensaio e calibração, sendo esta a base para a acreditação de tais laboratórios. Um dos requisitos técnicos abordados trata de métodos de ensaio e calibração, sendo estabelecido que laboratórios de ensaio devam aplicar procedimentos para a estimativa das incertezas de medição. ${ }^{3}$

Diante deste cenário, a estimativa da incerteza de medição em ensaios químicos torna-se de grande importância para a competência dos laboratórios de ensaios e, ainda, requisito para a confiabilidade do resultado analítico. De forma geral, o resultado de uma medição é somente uma aproximação ou estimativa de uma quantidade específica para um mensurando, assim, o resultado somente é completo quando acompanhado por uma declaração quantitativa de sua incerteza. ${ }^{4-6} \mathrm{O}$ Vocabulário Internacional de Metrologia - VIM define incerteza como um parâmetro não negativo que caracteriza a dispersão dos valores atribuídos a um mensurando, com base nas informações utilizadas. ${ }^{7}$

A abordagem tradicional para estimativa da incerteza de medição é baseada na identificação, quantificação e combinação de todas as

\footnotetext{
*e-mail: fabio.bazilio@incqs.fiocruz.br
}

contribuições individuais para a incerteza final. Nesta abordagem, chamada de bottom-up, a incerteza combinada final é derivada das incertezas dos componentes individuais, sendo adotada pela Organização Internacional de Normalização (ISO). ${ }^{8}$

A estimativa da incerteza do resultado de um ensaio perpassa pelo conhecimento dos pontos críticos do procedimento analítico, sendo necessário conhecer os fatores que podem vir a influenciar o seu resultado final. Alguns destes fatores principais envolvem preparo dos padrões analíticos, diluição das amostras, medições feitas em um equipamento e procedimento de quantificação por calibração. ${ }^{9} \mathrm{Com}$ as fontes de incerteza relevantes identificadas, através de ferramentas estatísticas, são estimadas as incertezas padrão atribuídas a cada fonte e a incerteza padrão combinada final.

Segundo o Guia para a Expressão da Incerteza de Medição GUM os componentes da incerteza são agrupados em duas categorias baseadas no seu método de avaliação, sendo elas "Tipo A" e "Tipo B". Incertezas do Tipo A são provenientes de análises estatísticas de uma série de repetições, enquanto incertezas do Tipo B são disponíveis a partir de quaisquer outros meios, sendo usualmente baseadas em julgamentos científicos utilizando-se de informações disponíveis. Cálculos de desvio padrão de média de uma série de observações como, por exemplo, repetitividade e utilização do método dos mínimos quadrados ordinários (MMQO) para ajuste de curva linear são exemplos típicos de incertezas do Tipo A. Por outro lado, incertezas obtidas em certificados de calibração são exemplos de incertezas do Tipo B. ${ }^{10,11}$

Apesar da dificuldade de compreensão e de domínio das ferramentas estatísticas necessárias para os cálculos envolvidos, atualmente são encontrados diversos guias orientativos nacionais e internacionais para facilitar o cálculo da incerteza dos ensaios. Nesses guias são encontradas metodologias de cálculos, cada uma mais adequada a um determinado tipo de ensaio. Exemplos de cálculos também são encontrados para auxiliar na compreensão dos métodos propostos. O Guia para a Quantificação de Incertezas em Ensaios Químicos (OGC007) disponibilizado pelo Instituto Português de Acreditação - IPAC é uma referência orientativa para o assunto. ${ }^{12} \mathrm{O}$ Instituto Nacional de Metrologia, Qualidade e Tecnologia - INMETRO disponibiliza também alguns documentos orientativos para auxiliar no processo de estimativa da incerteza de medição. 
Devido à crescente necessidade de garantia de resultados mais confiáveis e maior disponibilidade destes guias, observa-se um aumento do número de laboratórios que utilizam esta ferramenta na expressão de seus resultados analíticos. Contudo, na área de migração de resíduos de embalagens não são encontradas na literatura referências para a estimativa da incerteza de medição dos resultados analíticos.

O presente trabalho teve como objetivo apresentar um estudo de caso sobre a estimativa da incerteza de medição no ensaio de migração de $\varepsilon$-caprolactama da embalagem para alimentos gordurosos, com determinação por cromatografia a gás com detector por ionização em chama - DIC, demonstrando aspectos importantes do cálculo, assim como suas aplicações e um breve guia para o cálculo.

\section{PARTE EXPERIMENTAL}

O ensaio de migração consiste na análise da migração do monômero $\varepsilon$-caprolactama presente em embalagens contendo poliamida 6 para um solvente simulante (etanol 95\%) e posterior determinação por cromatografia a gás - CG com detecção por ionização em chama - DIC. Foi realizado no laboratório de alimentos e contaminantes orgânicos, do departamento de química do Instituto Nacional de Controle de Qualidade em Saúde - INCQS.

Para este ensaio foram cortadas três seções com $1 \mathrm{dm}^{2}$ de área do filme de poliamida. Cada seção foi colocada em contato com 100 $\mathrm{mL}$ de solvente simulante (etanol 95\%) em uma placa de petri com tampa, acondicionada em estufa a $60 \pm 3{ }^{\circ} \mathrm{C}$, durante 3 h e $30 \pm 10$ min. Após o período de contato cada solução foi transferida para um balão volumétrico de $100 \mathrm{~mL}$ e seu volume completado até a marca com etanol 95\%. ${ }^{1}$ A curva analítica foi construída com três padrões preparados a partir de uma solução estoque de $1000,8 \pm 3,0 \mathrm{mg} \mathrm{L}^{-1}$ do monômero, com um fator de abrangência $(k)$ para a incerteza expandida igual a 2,00. A Tabela 1 apresenta os volumes das alíquotas e os volumes finais para o preparo dos padrões da curva analítica.

Tabela 1. Volumes de preparo dos padrões da curva analítica

\begin{tabular}{ccc}
\hline Padrão & Volume da alíquota & Volume final \\
\hline$P_{1}$ & $(0,20 \pm 0,01) \mathrm{mL}, k=2,32$ & $(99,97 \pm 0,06) \mathrm{mL}, k=2,11$ \\
$P_{2}$ & $(1,69 \pm 0,01) \mathrm{mL}, k=2,32$ & $(99,93 \pm 0,06) \mathrm{mL}, k=2,11$ \\
$P_{3}$ & $(3,17 \pm 0,02) \mathrm{mL}, k=2,32$ & $(99,94 \pm 0,06) \mathrm{mL}, k=2,11$ \\
\hline
\end{tabular}

Os valores de $k(2,11$ e 2,32$)$ são característicos dos aparatos volumétricos utilizados, sendo estes valores disponíveis em seus respectivos certificados de calibração.

Após o preparo dos padrões de calibração, foram injetadas com o auxílio de um injetor automático modelo AOC-20i três alíquotas de $2 \mu \mathrm{L}$ de cada padrão e amostra obtida pelo ensaio de migração, em ordem aleatória, em um cromatógrafo a gás Shimadzu GC-2010 acoplado a um detector por ionização em chama (DIC), com coluna de sílica fundida recoberta internamente com fase estacionária constituída de 5\% fenilmetilsilicone (HP-5 da J \& W Scientific) com dimensões de $30 \mathrm{~m}$ de comprimento $\mathrm{x} 0,53 \mathrm{~mm}$ de d.i. $\mathrm{x} 2,65 \mu \mathrm{m}$ de espessura de filme interno. As temperaturas do injetor, coluna e detector foram, respectivamente, $210^{\circ} \mathrm{C} ; 100^{\circ} \mathrm{C}(1 \mathrm{~min})$ até $180^{\circ} \mathrm{C}(3 \mathrm{~min})$, com acréscimo de $4^{\circ} \mathrm{C} \min ^{-1} \mathrm{e} 250^{\circ} \mathrm{C}$ e a vazão do gás de arraste hélio foi $10,14 \mathrm{~mL} \mathrm{~min}{ }^{-1}$.

\section{Estimativa da incerteza de medição}

O processo de estimativa da incerteza é iniciado pela determinação do mensurando e identificação das fontes de incerteza para a metodologia analítica. Para cada fonte de incerteza as variáveis de entrada foram determinadas e as incertezas associadas a cada fonte, quantificadas. Foram identificadas possíveis correlações entre as variáveis e calcularam-se as incertezas combinada e expandida.
No ensaio de migração estudado, o mensurando foi representado pela concentração de migração do analito para o solvente simulante. A determinação da concentração de migração foi realizada através da Equação 1, proveniente da equação da curva analítica.

$$
C_{0}=\frac{A-a}{b}
$$

onde, $A$ é a média das áreas dos picos referentes às amostras obtidas pelo ensaio de migração, $C_{0}$ a concentração média do analito encontrada na amostra, $b$ o coeficiente angular da reta, e $a$ o coeficiente linear.

É necessário que a estimativa da incerteza de medição seja desenvolvida de maneira completa e confiável, observando-se a contribuição quantitativa de cada fonte. ${ }^{6}$ Devem ser considerados os erros que possam ocorrer durante o processo analítico, desde o preparo dos padrões até a obtenção do resultado final. ${ }^{13}$ Através de uma análise detalhada da expressão do mensurando e do conhecimento dos pontos críticos do ensaio, é possível identificar as fontes de incerteza que possam vir a impactar no resultado final. A Figura 1 apresenta as fontes de incerteza mais importantes. ${ }^{14}$

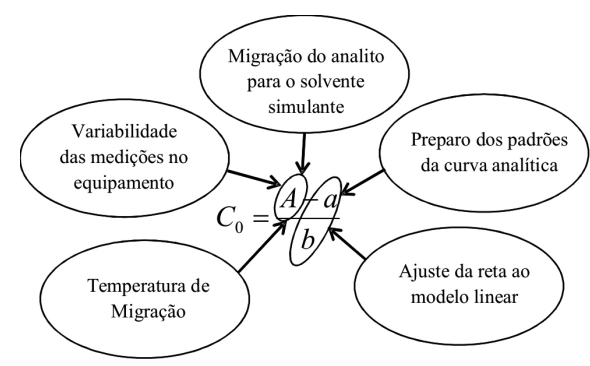

Figura 1. Incertezas de maior impacto para o resultado final

Para uma estimativa adequada da incerteza de medição, é importante que o processo analítico seja cuidadosamente observado. No entanto, deve ser evitada a duplicidade de contribuição das incertezas, o que poderia causar uma superestimativa da incerteza combinada. Com este fim, foi construído um diagrama de causa e efeito, também conhecido como diagrama de Ishikawa ou espinha de peixe. ${ }^{15}$ A Figura 2 apresenta o diagrama de causa e efeito construído para o ensaio de migração. O diagrama evidencia as principais fontes de incerteza identificadas para o ensaio.

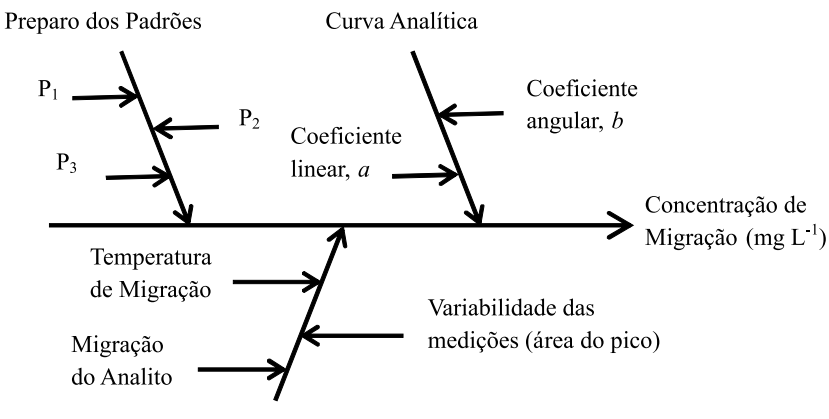

Repetitividade (área do pico)

Figura 2. Diagrama de causa e efeito para o ensaio de migração

As principais fontes de incerteza identificadas para o ensaio de migração foram: incerteza associada à curva analítica $\left(u_{(C O)}\right)$, incerteza associada à repetitividade da metodologia $\left(u_{(\text {rep })}\right)$ e incerteza associada ao preparo dos padrões da curva analítica $\left(u_{(d i l)}\right)$.

Incerteza associada ao preparo dos padrões de calibração $u_{(d i l)}$ A incerteza de medição associada ao preparo dos padrões $u_{(d i l)}$ 
engloba as incertezas associadas ao preparo de cada padrão $\left(u_{(p l)}\right.$, $\left.u_{(p 2)}, u_{(p 3)}\right)$ sendo uma incerteza combinada destas. ${ }^{9,12}$ As incertezas associadas ao preparo de cada padrão $u_{(P n)}$ foram calculadas através dos coeficientes de sensibilidade de cada variável e suas relações matemáticas, como apresentado na Equação 2.

$$
u_{\left(P_{n}\right)}=\sqrt{\left(\frac{C_{e} \times u_{\left(v_{e}\right)}}{v_{p}}\right)^{2}+\left(\frac{C_{e} \times v_{e} \times u_{\left(v_{p}\right)}}{v_{p}{ }^{2}}\right)^{2}+\left(\frac{v_{e} \times u_{\left(C_{e}\right)}}{v_{p}}\right)^{2}}
$$

onde, $C_{e}$ é a concentração da solução estoque, $v_{e}$ o volume da alíquota da solução estoque, $v_{p}$ o volume final da solução do padrão de calibração, $u_{\left(P_{n}\right)}$ a incerteza associada à solução padrão, $u_{\left(v_{e}\right)}$ a incerteza associada à alíquota da solução estoque, $u_{\left(v_{p}\right)}$ a incerteza associada ao volume final da solução do padrão de calibração e $u_{\left(C_{e}\right)}$ a incerteza associada à concentração da solução estoque.

Estimadas as incertezas de cada padrão preparado para a curva analítica, estas são combinadas através da Equação 3.

$$
u_{(d i l)}=\sqrt{\left(\frac{u_{\left(P_{1}\right)}}{C_{P_{1}}}\right)^{2}+\left(\frac{u_{\left(P_{2}\right)}}{C_{P_{2}}}\right)^{2}+\left(\frac{u_{\left(P_{3}\right)}}{C_{P_{3}}}\right)^{2}}
$$

onde, $C_{P_{n}}$ é a concentração de cada $n$ solução de trabalho e $u_{\left(P_{n}\right)}$ a incerteza associada ao preparo de cada $n$ solução de trabalho.

\section{Incerteza associada à curva analítica $u_{\left(C_{0}\right)}$}

A incerteza associada à interpolação na curva analítica $u_{\left(C_{0}\right)}$ é baseada no cálculo do intervalo de confiança da curva construída através do método dos mínimos quadrados ordinários (MMQO), considerando-se o modelo de resposta linear. ${ }^{9} \mathrm{~A}$ incerteza associada à curva analítica é estimada através da Equação 4.

$$
u_{\left(C_{0}\right)}=\frac{S_{\text {residual }}}{b} \times \sqrt{\frac{1}{p}+\frac{1}{n}+\frac{\left(C_{0}-\bar{C}\right)^{2}}{S_{x x}}}
$$

onde, $S_{\text {residual }}=\sqrt{\frac{\sum_{j=1}^{n}\left[A_{j}-A_{E s p}\right]^{2}}{n-2}}, S_{x x}=\sum_{j=1}^{n}\left(C_{j}-\bar{C}\right)^{2}, C_{0}$ é a concentração da solução de migração encontrada para a amostra, $p$ o número de repetições para determinar $C_{0}, n$ o número total de determinações para construção da curva analítica, $\bar{C}$ a concentração média da curva analítica, e $b$ a inclinação da reta.

\section{Incerteza associada à repetitividade $u_{(\text {rep })}$}

A incerteza associada à repetitividade é oriunda de efeitos aleatórios e é, em geral, medida a partir de experimentos repetidos e quantificada em termos de desvio padrão de resposta. ${ }^{9}$ No ensaio de migração são realizadas três repetições da amostra ( 3 ensaios independentes) e ao final do ensaio uma alíquota de cada uma das três soluções genuínas é analisada por CG-DIC, determinando-se a sua concentração através da curva analítica preparada. O resultado final da migração é reportado através da média das áreas dos picos cromatográficos das três soluções de migração. A incerteza associada à repetitividade da metodologia $u_{(\text {rep })}$ provém do desvio padrão das áreas dos picos calculadas para as três soluções e engloba as variações inerentes ao processo analítico. A incerteza associada à repetitividade é estimada através da Equação 5. ${ }^{16}$

$$
u_{(\text {rep })}=\frac{S_{\text {area }}}{\sqrt{p}} \times \frac{1}{b}
$$

onde, $S_{\text {area }}$ é o desvio padrão das áreas dos picos correspondentes às alíquotas das soluções de migração das repetições, $p$ o número de repetições para determinar a concentração de migração para a amostra e $b$ o coeficiente angular da curva analítica preparada.

\section{Estimativa da incerteza combinada final $u_{\text {Comb }}$}

A incerteza padrão combinada final $u_{\text {Comb }}$ associada ao mensurando, sendo este o resultado da medição analítica, é obtida pela combinação apropriada das incertezas padrão estimadas para as grandezas de entrada. ${ }^{9}{ }^{10} \mathrm{~A}$ incerteza combinada final, para o ensaio de migração estudado, é estimada através da Equação 6.

$$
u_{C o m b}=C_{0} \times \sqrt{\left(u_{(d i l)}\right)^{2}+\left(\frac{u_{(r e p)}}{C_{0}}\right)^{2}+\left(\frac{u_{\left(C_{0}\right)}}{C_{0}}\right)^{2}}
$$

\section{Estimativa da incerteza expandida $U$}

Através da incerteza combinada final é calculada a incerteza expandida associada ao resultado analítico. A incerteza expandida é uma medida da incerteza, dada para uma função de distribuição de probabilidade apropriada ao resultado, habitualmente distribuição normal, associada a um nível elevado de confiança. A incerteza expandida é dada pelo produto entre a incerteza padrão combinada estimada e um fator de abrangência $k$ para um nível de confiança desejado (Equação 7). 5,7,12

$$
U=k \times u_{(\mathrm{Comb})}
$$

onde, $k$ é o fator de abrangência.

Nos casos em que a incerteza $u_{\text {comb }}(y)$ é aproximadamente normal ou os graus de liberdade efetivos $v_{e f f}$ de $u_{\text {comb }}(y)$ são de tamanho significativo, utiliza-se $k=2$ para um nível de confiança de aproximadamente $95 \%$. Para quase todos os fins, devido à natureza aproximativa do processo de estimativa da incerteza de medição, pode-se adotar $k=$ 2. ${ }^{9,10}$ Contudo, é possível também determinar o valor de $k$, quando o processo de estimativa de incerteza não possui graus de liberdade efetivos suficientemente grandes. Segundo o ISO GUM, os graus de liberdade efetivos podem ser calculados através da fórmula WelchSatterthwaite (Equação 8). ${ }^{10}$

$$
v_{e f f}=\frac{u_{c}^{4}(y)}{\sum_{i=1}^{N} \frac{u_{i}^{4}(y)}{v_{i}}}
$$

onde, $u_{c}(y)$ é a incerteza combinada da metodologia; $u_{i}(y)$ é a incerteza padrão da componente $i$ e $v_{i}$ é o número de graus de liberdade da componente $i$.

\section{RESULTADOS E DISCUSSÃO}

A construção da curva analítica foi realizada com as áreas dos picos cromatográficos referentes aos padrões analisados. Através do método dos mínimos quadrados ordinários, foi realizado o ajuste da curva ao modelo linear. A Equação 9 representa a reta ajustada para a curva analítica preparada.

$$
Y=17207,97 X+9609,93
$$

com, $S_{x x}=1326,90$ e $S_{\text {residual }}=27470,25$.

A concentração de migração de $\varepsilon$-caprolactama encontrada para a amostra analisada foi de $13,0 \mathrm{mg} \mathrm{L}^{-1}$. Essa concentração foi determinada através da interpolação da média das áreas dos picos das três repetições genuínas na curva analítica. Na Tabela 2 são apresentados os resultados do ensaio de migração. 
Tabela 2. Resultados do ensaio de migração

\begin{tabular}{cccc}
\hline Solução de migração & Área & Concentração $\left(\mathrm{mg} \mathrm{L}^{-1}\right)$ & $S_{\text {area }}$ \\
\hline Repetição 1 & 214487 & 11,91 & \\
Repetição 2 & 232517 & 12,95 & 19994,69 \\
Repetição 3 & 254414 & 14,23 & \\
\hline
\end{tabular}

$S_{\text {area }}$, desvio padrão das áreas dos picos referentes às repetições.

A incerteza combinada estimada para a concentração de migração de $\varepsilon$-caprolactama determinada para a amostra ensaiada foi de $1,3 \mathrm{mg} \mathrm{L}^{-1}$. Na Tabela 3 são apresentados os valores calculados para as incertezas padrão e suas respectivas incertezas padrão relativas.

O gráfico comparativo das contribuições das fontes de incerteza do ensaio (Figura 3) é uma ferramenta para a identificação das fontes de maior impacto na incerteza padrão combinada final. Ajustes na metodologia podem ser realizados para minimizar a contribuição destas fontes.

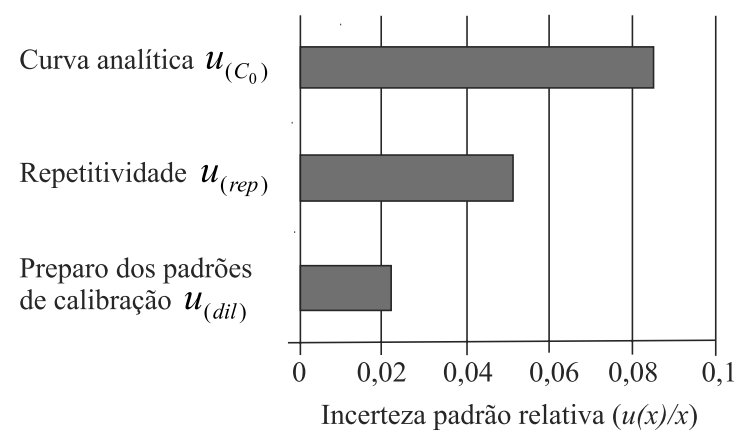

Figura 3. Contribuições das fontes de incerteza do ensaio para a incerteza combinada

A incerteza proveniente do preparo dos padrões, estimada para o ensaio de migração, foi a de menor contribuição para a incerteza combinada final, enquanto a incerteza associada à curva analítica foi a de maior impacto. A incerteza associada ao preparo dos padrões da curva analítica é proveniente das incertezas relacionadas aos volumes de diluição dos padrões e da incerteza associada à concentração da solução concentrada. Sendo assim, espera-se uma incerteza de baixa contribuição.

Para o ensaio de migração estudado, o número de graus de liberdade efetivos calculados através da fórmula Welch-Satterthwaite foi 9 e a incerteza expandida estimada foi $3,0 \mathrm{mg} \mathrm{L}^{-1}$ para um fator de abrangência $k=2,32$, com um nível de confiança de 95,45\%. A concentração de migração de $\varepsilon$-caprolactama para a amostra ensaiada foi reportada, segundo recomendações do ISO GUM, na forma de: Concentração $=(13,0 \pm 3,0) \mathrm{mg} \mathrm{L}^{-1}$.

Os resultados encontrados e a metodologia empregada para o cálculo da incerteza se mostraram satisfatórios. A incerteza expandida final calculada, cerca de $23 \%$ da concentração da amostra, é compatível com a metodologia analítica adotada para o ensaio. A Comissão do Codex Alimentarius, criada em 1963 pela Organização das Nações Unidas para Agricultura e Alimentação - FAO e Organização Mundial de Saúde OMS, tem como principais objetivos proteger a saúde dos consumidores, assegurando justas práticas comerciais no comércio internacional de alimentos. A Comissão estabelece no documento Guidelines on Measurement Uncertainty (CAC/GL 54-2004) valores de incerteza expandida esperados para ensaios químicos a serem reportados em ensaios colaborativos. Para concentrações da ordem de $10 \mathrm{mg} \mathrm{kg}^{-1}$ é esperada uma incerteza expandida de aproximadamente $22 \% .{ }^{17}$

\section{CONCLUSÃO}

Através dos guias orientativos, como EURACHEM/CITAC e ISO GUM, foi possível desenvolver uma metodologia para a estimativa da incerteza de medição do ensaio e estimá-la para a amostra ensaiada. A incerteza expandida estimada foi considerada satisfatória, levando-se em consideração os valores estabelecidos pela comissão do Codex Alimentarius para a faixa de concentração de migração encontrada para a amostra.

Tabela 3. Incertezas padrão estimadas para o ensaio de migração

\begin{tabular}{lccccc}
\hline Fonte de incerteza & Classificação da componente & $v$ & Valor $x$ & $u(x)$ & $u(x) / x$ \\
\hline Repetitividade $u_{(\text {rep })}$ & Tipo A & 2 & $13,0 \mathrm{mg} \mathrm{L}^{-1}$ & $0,67 \mathrm{mg} \mathrm{L}^{-1}$ & 0,051 \\
Curva analítica $u_{\left(C_{0}\right)}$ & Tipo A & 7 & $13,0 \mathrm{mg} \mathrm{L}^{-1}$ & $1,1 \mathrm{mg} \mathrm{L}^{-1}$ & 0,085 \\
Diluição dos Padrões $u_{(\text {dil })}$ & Tipo B & $\infty$ & 1 & 0,022 & 0,022 \\
\hline
\end{tabular}

$v$, graus de liberdade da componente; $u(x)$, incerteza padrão; $u(x) / x$, incerteza padrão relativa.

\section{REFERÊNCIAS}

1. Bomfim, M. V. J.; Abrantes, S. M. P.; Zamith, H. P. S.; Quim. Nova 2010, 33, 1114.

2. Brasil, Resolução n ${ }^{\circ} 105$ de 19 de maio de 1999, Diário Oficial da República Federativa do Brasil, Brasília, DF, 20 de maio de 1999.

3. Associação Brasileira de Normas Técnicas, NBR ISO/IEC 17025; Requisitos gerais para a competência de laboratórios de ensaio e calibração, 2a ed., Rio de Janeiro, 2005.

4. National Institute of Standards and Technology, Technical Note 1297; Guidelines for Evaluating and Expressing the Uncertainty of NIST Measurement Results, 1994 ed., Washington, 1994.

5. Oliveira, E. C.; Aguiar, P. F.; Quim. Nova 2009, 32, 1655.

6. de la Cruz, M. H. C.; Rodrigues, J. M.; Couto, P. R. G.; Cunha, V. S.; Bremser, W.; Quim. Nova 2010, 33, 1578.

7. Instituto Nacional de Metrologia, Qualidade e Tecnologia; Vocabulário Internacional de Metrologia: Conceitos Fundamentais e Gerais e Termos Associados (VIM 2008), 1ª ed., Rio de Janeiro, 2009.
8. Tomic, T.; Nasipak, N. U.; Babic, S.; Accred. Qual. Assur. (2012), doi:10.1007/s00769-011-0872-0.

9. EURACHEM/CITAC Working Group; Quantifying Uncertainty in Analytical Measurement, $2^{\text {nd }}$ ed., London, 2000.

10. Associação Brasileira de Normas Técnicas - Instituto Nacional de Metrologia, Qualidade e Tecnologia; Guia para a Expressão da Incerteza de Medição, $3^{\text {a }}$ ed., Rio de Janeiro, 2003.

11. Priel, M.; Accred. Qual. Assur. 2009, 14, 235.

12. Instituto Português de Acreditação, OGC007; Guia para a Quantificação de Incerteza em Ensaios Químicos, Caparica, 2007.

13. Shegunova, P.; Bercaru, O.; Sejeroe-Olsen, B.; Accred. Qual. Assur. 2008, 13, 11.

14. Leito, S.; Leito, I.; Accred. Qual. Assur. 2004, 9, 666.

15. Buchmann, J. H.; Sarkis, J. E. S.; Quim. Nova 2002, 25, 111.

16. Ávila, A. K.; Araújo, T. O.; Couto, P. R. G.; Borges, R. M. H.; Analytica 2004, 11, 48 .

17. FAO/WHO Codex Alimentarius Commission, CAC/GL 54-2004; Guidelines on Measurement Uncertainty, rev. 1, Rome, 2011. 